\title{
Tree spanners on chordal graphs: complexity and algorithms
}

\author{
Andreas Brandstädt ${ }^{\mathrm{a}}$, Feodor F. Dragan ${ }^{\mathrm{b}, *}$, Hoàng-Oanh Le $\mathrm{e}^{\mathrm{a}, 1}$, \\ Van Bang Le ${ }^{a}$ \\ ${ }^{a}$ Institut für Theoretische Informatik, Fachbereich Informatik, Universität Rostock, \\ 18051 Rostock, Germany \\ ${ }^{\mathrm{b}}$ Department of Computer Science, Kent State University, Kent, OH 44242, USA
}

Received 10 September 2002; received in revised form 17 July 2003; accepted 28 July 2003 Communicated by G.F. Italiano

\begin{abstract}
A tree t-spanner $T$ in a graph $G$ is a spanning tree of $G$ such that the distance in $T$ between every pair of vertices is at most $t$ times their distance in $G$. The TREE $t$-SPANNER problem asks whether a graph admits a tree $t$-spanner, given $t$. We substantially strengthen the hardness result of Cai and Corneil (SIAM J. Discrete Math. 8 (1995) 359-387) by showing that, for any $t \geqslant 4$, TREe $t$-SPANNER is NP-complete even on chordal graphs of diameter at most $t+1$ (if $t$ is even), respectively, at most $t+2$ (if $t$ is odd). Then we point out that every chordal graph of diameter at most $t-1$ (respectively, $t-2$ ) admits a tree $t$-spanner whenever $t \geqslant 2$ is even (respectively, $t \geqslant 3$ is odd), and such a tree spanner can be constructed in linear time.

The complexity status of TREE 3-SPANNER still remains open for chordal graphs, even on the subclass of undirected path graphs that are strongly chordal as well. For other important subclasses of chordal graphs, such as very strongly chordal graphs (containing all interval graphs), 1-split graphs (containing all split graphs) and chordal graphs of diameter at most 2, we are able to decide TREE 3-SPANNER efficiently.
\end{abstract}

(c) 2003 Elsevier B.V. All rights reserved.

Keywords: Tree spanners; Spanners; Chordal graphs; Distance; Spanning trees; NP-completeness; Efficient graph algorithms

\footnotetext{
${ }^{2}$ An extended abstract of this paper appeared in the proceedings of The 13th Annual International Symposium on Algorithms and Computation (ISAAC 2002), November 20-23, 2002, Vancouver, Canada, Springer, Lecture Notes in Computer Science 2518, pp. 163-174.

* Corresponding author. Tel.: +1-330-6729058; fax: +1-330-6727824.

E-mail addresses: ab@informatik.uni-rostock.de (A. Brandstädt), dragan@cs.kent.edu (F.F. Dragan), hoang-oanh.le@informatik.uni-rostock.de (H.-O. Le), le@informatik.uni-rostock.de (V.B. Le).

${ }^{1}$ Research of this author supported by DFG, Project no. Br1446-4/1.
} 


\section{Introduction and results}

All graphs considered are connected. For two vertices in a graph $G, d_{G}(x, y)$ denotes the distance between $x$ and $y$; that is, the number of edges in a shortest path in $G$ joining $x$ and $y$. The value $\operatorname{diam}(G):=\max d_{G}(x, y)$ is the diameter of the graph $G$.

Let $t \geqslant 2$ be a fixed integer. A spanning tree $T$ of a graph $G$ is a tree $t$-spanner of $G$ if and only if, for every pair of vertices $x, y$ of $G, d_{T}(x, y) \leqslant t \cdot d_{G}(x, y)$. Tree $t$-Spanner is the following problem: Given a graph $G$, does $G$ admit a tree $t$-spanner?

There are many applications of tree spanners in different areas; especially in distributed systems and communication networks. In [1], for example, it was shown that tree spanners can be used as models for broadcast operations; see also [25]. Moreover, tree spanners also appear in biology [2], and in [29], tree spanners were used in approximating the bandwidth of graphs. We refer to $[8,7,26,28]$ for more background information on tree spanners.

In [7] Cai and Corneil gave a linear time algorithm solving TREe 2-SPANNER and proved that TREe $t$-SPANNER is NP-complete for any $t \geqslant 4$. A graph is chordal if it does not contain any chordless cycle of length at least four. For a popular subclass of chordal graph, the strongly chordal graphs, Brandstädt et al. [3] proved that, for every $t \geqslant 4$, TREe $t$-SPAnNer is solvable in linear time. Indeed, they show that every strongly chordal graph admits a tree 4-spanner. In contrast, one of our results is

Theorem 1. For any $t \geqslant 4$, TREe $t$-SPanneR is NP-complete on chordal graphs of diameter at most $t+1$ (if $t$ is even), respectively, of at most $t+2$ (if $t$ is odd).

Comparing with a recent result due to Papoutsakis [24], it is interesting to note that the union of two tree $t$-spanners, $t \geqslant 4$, may contain chordless cycles of any length, while, for $t=3$, the graph being the union of any two tree 3-spanners of a graph may contain even chordless cycles of any length but it cannot contain any odd chordless cycle other than a triangle [24]. This perhaps indicates the difficulty in proving Theorem 1 and in treating the $t=3$ case. Indeed, our reduction from 3SAT to TREE $t$-SPANNER given in Section 2 is quite involved.

Moreover, to the best of our knowledge, Theorem 1 is the first hardness result for Tree $t$-Spanner on a restricted, well-understood graph class. Notice that in [16] it is shown that Tree $t$-Spanner, $t \geqslant 4$, is NP-complete on planar graphs if the integer $t$ is part of the input.

In view of the diameter constraints in Theorem 1, we note that Tree $t$-Spanner remains open on chordal graphs of diameter $t$ ( $t$ is even) and of diameter $t-1, t$ or $t+1$ (if $t$ is odd). For "smaller" diameter we have

Theorem 2. For any even integer $t$, every chordal graph of diameter at most $t-1$ admits a tree t-spanner, and such a tree spanner can be constructed in linear time. For any odd integer $t$, every chordal graph of diameter at most $t-2$ admits a tree $t$-spanner, and such a tree spanner can be constructed in linear time. 
We were also able to show that chordal graphs of diameter at most $t-1$ ( $t$ is odd) admit tree $t$-spanners if and only if chordal graphs of diameter 2 admit tree 3 -spanners. This result is used to show that every chordal graph of diameter at most $t-1$ ( $t$ is odd), which is a planar graph or a $k$-tree, for $k \leqslant 3$, has a tree $t$-spanner, while such a tree spanner can be constructed in polynomial time. Note that, for any fixed $t$, there is a 2-tree without a tree $t$-spanner [20]. So, even those kind of results are of interest. Unfortunately, the reduction above (from arbitrary odd $t$ to $t=3$ ) is of no direct use for general chordal graphs because not every chordal graph of diameter at most 2 admits a tree 3-spanner. One of our theorems characterizes those chordal graphs of diameter at most 2 that admit such spanners.

We now discuss Tree $t$-Spanner on important subclasses of chordal graphs. It is well-known that chordal graphs are exactly the intersection graphs of subtrees in a tree $[5,17,31]$. Thus, intersection graphs of paths in a tree, called path graphs, form a natural subclass of chordal graphs. TREE $t$-SPANNER remains unresolved even on this natural subclass of chordal graphs.

The complexity status of TREE 3-SPANNER remains a long standing open problem. However, it can be solved efficiently for many particular graph classes, such as cographs and complements of bipartite graphs [6], directed path graphs [21] (hence for all interval graphs [20,21,27]), split graphs [6,20,29], permutation graphs and regular bipartite graphs [22], convex bipartite graphs [29], and recently for planar graphs [16]. In $[6,23,24]$, some properties of graphs admitting a tree 3 -spanner are discussed.

On chordal graphs, however, TREE 3-SPANNER remains open even on path graphs which are strongly chordal as well. For some important subclasses of chordal graphs we can decide Tree 3-Spanner efficiently. Graphs considered in the theorem below are defined in Sections 5 and 6.

Theorem 3. All very strongly chordal graphs and all 1-split graphs admit a tree 3-spanner, and such a tree 3-spanner can be constructed in linear time.

Theorem 4. For a given chordal graph $G=(V, E)$ of diameter at most 2, TREe 3-SPANNER can be decided in $O(|V||E|)$ time. Moreover, a tree 3-spanner of $G$, if it exists, can be constructed within the same time bound.

Theorem 3 improves previous results on tree 3-spanners in interval graphs [20,22,27] and on split graphs $[6,20,29]$. The complexity status of TREE $t$-SPANNER on chordal graphs considered in this paper is summarized in Table 1 and Fig. 1.

Notations and definitions not given here may be found in any standard textbook on graphs and algorithms. We write $x y$ for the edge joining vertices $x, y ; x$ and $y$ are also called endvertices of $x y$. For a set $C$ of vertices, $N(C)$ denotes the set of all vertices outside $C$ adjacent to a vertex in $C ; N(x)$ stands for $N(\{x\})$ and $\operatorname{deg}(x)$ stands for $|N(x)|$. We set $d(v, C):=\min \{d(v, x): x \in C\}$. The eccentricity of a vertex $v$ in $G$ is the maximum distance from $v$ to other vertices in $G$. The radius $r(G)$ of $G$ is the minimum of all eccentricities and the diameter $\operatorname{diam}(G)$ of $G$ is the maximum of all eccentricities. A cutset of a graph is a set of vertices whose deletion disconnects the graph. A graph is non-separable if it has no one-element cutset, and triconnected 
Table 1

The complexity status of TREE $t$-SPANNER on chordal graphs under diameter constraints.

\begin{tabular}{ll}
\hline Diameter at most & Complexity \\
\hline$t+2, t \geqslant 5$ odd & NP-complete \\
$t+1, t \geqslant 4$ even & NP-complete \\
$t+1, t \geqslant 3$ odd & $?$ \\
$t, t \geqslant 3$ & $?$ \\
$t-1, t \geqslant 5$ odd & $?$ \\
$t-1, t=3$ & polynomial time \\
$t-1, t \geqslant 2$ even & linear time \\
$t-2, t \geqslant 3$ odd & linear time \\
\hline
\end{tabular}

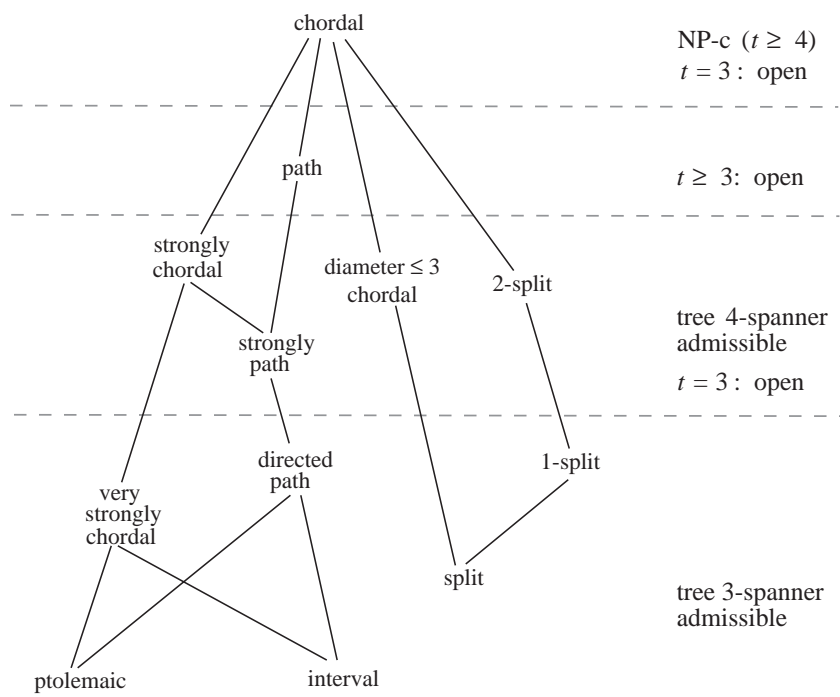

Fig. 1. The complexity status of TREE $t$-SPANNER on chordal graphs and important subclasses.

if it has no cutset with $\leqslant 2$ vertices. Blocks in a graph are maximal non-separable subgraphs of that graph.

Clearly, a graph contains a tree $t$-spanner if and only if each of its blocks contains a tree $t$-spanner. Note also that dividing a graph into blocks can be done in linear time. Thus, in this paper, we consider non-separable graphs only. Graphs having a tree $t$-spanner are called tree $t$-spanner admissible.

Finally, we will use the following fact in checking whether a spanning tree is a tree $t$-spanner.

Proposition 1 (Cai and Corneil [7]). A spanning tree $T$ of a graph $G$ is a tree $t$-spanner if and only if, for every edge $x y$ of $G, d_{T}(x, y) \leqslant t$. 


\section{NP-completeness, $t \geqslant 4$}

In this section we will show that, for any fixed $t \geqslant 4$, TREE $t$-SPANNER is NP-complete on chordal graphs. The proof is a reduction from 3SAT, for which the following family of chordal graphs will play an important role.

First, $S_{1}[x, y]$ stands for a triangle with two labeled vertices $x$ and $y$. Next, for a fixed integer $k \geqslant 1, S_{k+1}[x, y]$ is obtained from $S_{k}[x, y]$ by taking a new vertex $v_{e}$ for every edge $e \neq x y$ in $S_{k}[x, y]$ that belongs to exactly one triangle and joining $v_{e}$ to exactly the two endvertices of $e$. We write also $S_{k}$ for $S_{k}[x, y]$ for some suitable labeled vertices $x, y$. See Fig. 2.

Equivalently, $S_{k+1}[x, y]$ is obtained from two disjoint $S_{k}[a, b]$ and $S_{k}[c, d]$ by identifying the two vertices $b, d$ to a vertex $z$ and joining the vertices $x:=a$ and $y:=c$ by an edge. With this notation, $z$ is the common neighbor of $x$ and $y$ in $S_{k+1}[x, y]$, and we call $S_{k}[x, z]$ and $S_{k}[y, z]$ the two corresponding $S_{k}$ in $S_{k+1}[x, y]$. We denote by $S_{k}[x, y]$ the graph $S_{k}[x, y]-y$, that is, the graph obtained from $S_{k}[x, y]$ by deleting the vertex $y$. The following observations collect basic facts on $S_{k}$ used in the reduction later.

\section{Observation 1.}

(1) For every $v \in S_{k}[x, y], d_{S_{k}[x, y]}(v,\{x, y\}) \leqslant\lceil k / 2\rceil$,

(2) $S_{k}[x, y]$ has a tree $(k+1)$-spanner containing the edge $x y$,

(3) $S_{k}[x, y)$ has a tree $k$-spanner $T$ such that, for each neighbor $y^{\prime}$ of $y$ in $S_{k}[x, y)$, $d_{T}\left(x, y^{\prime}\right) \leqslant k$.

Proof. (1) By induction on $k$. The statement is clearly true for $k=1,2$. Assume $k \geqslant 3$, and let $z_{1}$ be the common neighbor of $x, y$ in $S_{k}[x, y]$, let $z_{2}$ and $z_{3}$ be the common neighbor of $x, z_{1}$, respectively, of $y, z_{1}$ in the corresponding $S_{k-1}\left[x, z_{1}\right]$ and $S_{k-1}\left[y, z_{1}\right]$. By symmetry, we may assume that $v \in S_{k-2}\left[x, z_{2}\right]=: A$ or $v \in S_{k-2}\left[z_{1}, z_{2}\right]=: B$.

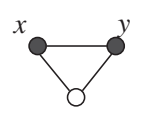

$S_{1}[x ; y]$

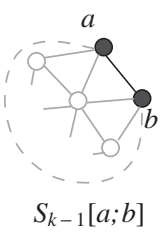

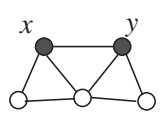

$S_{2}[x ; y]$

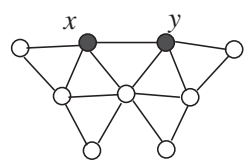

$S_{3}[x ; y]$

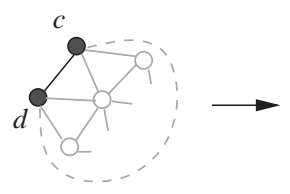

$S_{k-1}[c ; d]$

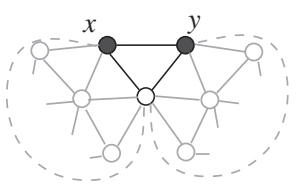

$S_{k}[x ; y]$

Fig. 2. The graph $S_{k}[x, y]$ obtained from $S_{k-1}[a, b]$ and $S_{k-1}[c, d]$ by identifying $b=d$ and joining $x=a$ with $y=c$. 


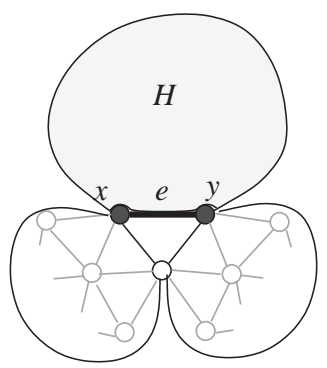

Fig. 3. The graph obtained from $H$ and $S_{k}[x, y]$ by identifying the edge $e=x y$.

Consider the case $v \in A$. The induction hypothesis gives us that

$$
d_{A}(v, x) \leqslant\left\lceil\frac{k-2}{2}\right\rceil \text { or } \quad d_{A}\left(v, z_{2}\right) \leqslant\left\lceil\frac{k-2}{2}\right\rceil .
$$

In the first case we are done. In the second case, we have

$$
d_{S_{k}[x, y]}(v, x) \leqslant d_{A}\left(v, z_{2}\right)+1 \leqslant\left\lceil\frac{k-2}{2}\right\rceil+1 \leqslant\left\lceil\frac{k}{2}\right\rceil,
$$

and the statement follows. The case $x \in B$ is similar.

(2) By induction on $k$. The statement is clearly true for $k=1$. Assume $k \geqslant 2$, and let $z$ be the common neighbor of $x, y$ in $S_{k}[x, y]$. By the induction hypothesis, the corresponding $S_{k-1}[x, z]$ and $S_{k-1}[y, z]$ have a tree $k$-spanner $T_{1}$, respectively, $T_{2}$ containing the edge $x z$, respectively, $y z$. Then it is easy to see that $T:=T_{1} \cup\left(T_{2}-y z\right)+x y$ is a desired tree $(k+1)$-spanner of $G$.

(3) This is clear for $k=1,2$. Let $k \geqslant 3$ and let $z$ be the common neighbor of $x, y$ in $S_{k}[x, y]$, and number the $k$ neighbors of $y$ in the corresponding $S_{k-1}[y, z]$ by $z_{1}=z$, $z_{2}, \ldots, z_{k}$ such that $S_{k}[x, y)$ consists of $S_{k-1}\left[x, z_{1}\right], S_{k-2}\left[z_{1}, z_{2}\right], \ldots, S_{1}\left[z_{k-2}, z_{k-1}\right]$ and the edge $z_{k-1} z_{k}$. By (2), each of these graphs contains a tree $k$-spanner. The union of all these tree spanners plus the edge $z_{k-1} z_{k}$ gives us a tree $k$-spanner $T$ of $S_{k}[x, y)$ with $d_{T}\left(x, z_{i}\right)=i \leqslant k$.

Observation 2. Let $H$ be an arbitrary graph and let e be an arbitrary edge of $H$. Let $K$ be an $S_{k}[x, y]$ disjoint from $H$. Let $G$ be the graph obtained from $H$ and $K$ by identifying the edges $e$ and $x y$; see Fig. 3. Suppose that $T$ is a tree t-spanner in $G, t>k$, such that the $x y$-path $P$ in $T$ belongs to $H$. Then

(1) $d_{T}(x, y) \leqslant t-k$, and

(2) there exists an edge $u v \in K$ with $d_{T}(u, v) \geqslant d_{T}(x, y)+k$.

Proof. By induction on $k$. For $k=1$, the statements follows directly from the fact that $T$ is a tree $t$-spanner of $G$. Let $k>1$, and assume that the statements (1) and (2) are true for arbitrary $H$ and $S_{k-1}$.

Let $z$ be the common neighbor of $x$ and $y$ in $K$, and consider the $x z$-path $Q$ in $T$. As $P \subseteq H$ and $\{x, y\}$ is a cutset of $G, Q \subseteq K \backslash\{y\}$, say. Let $L$ and $R$ be the two 
corresponding $S_{k-1}[x, z]$, respectively, $S_{k-1}[y, z]$ of $K$. As $\{x, z\}$ is a cutset of $K$, $Q \subseteq L$. Hence the $y z$-path $P \cup Q$ in $T$ belongs to the graph $H^{\prime}$ induced by $H$ and $L$. The induction hypothesis, applied to $H^{\prime}$ and $R$, gives us that

$$
d_{T}(y, z) \leqslant t-(k-1) \text { and } d_{T}(u, v) \geqslant d_{T}(z, y)+(k-1)
$$

for some edge $u v \in R$, implying

$$
d_{T}(x, y)=d_{T}(y, z)-d_{T}(x, z) \leqslant t-(k-1)-1=t-k,
$$

and

$$
d_{T}(u, v) \geqslant d_{T}(z, y)+(k-1) \geqslant d_{T}(x, y)+d_{T}(x, z)+(k-1) \geqslant d_{T}(x, y)+1,
$$

for the edge $u v \in R \subset K$.

Part (1) of Observation 2 indicates a way to force an edge $x y$ to be a tree edge, or to force a path of the tree to belong to certain part of the graph: Choosing $k=t-1$ shows that $x y$ must be an edge of $T$, or else the $x y$-path in $T$ must belong to the part $S_{t-1}[x, y]$.

We now describe the reduction. Let $F$ be a 3SAT formula with $m$ clauses $C_{j}=$ $\left(c_{j 1}, c_{j 2}, c_{j 3}\right)$ over $n$ variables $v_{i}$. Set $\ell:=\lfloor t / 2\rfloor-2$ and $\lambda:=\lceil\ell / 2\rceil$. Since $t \geqslant 4, \ell \geqslant 0$ and $\lambda \geqslant 0$.

For each variable $v_{i}$ create the graph $G\left(v_{i}\right)$ as follows.

- Set $q_{i}^{0}:=v_{i}, q_{i}^{\ell+1}:=\overline{v_{i}}$. We will use $u \in\left\{q_{i}^{0}, q_{i}^{\ell+1}\right\}$ as a vertex in our graph as well as a literal in the given 3SAT formula $F$.

- Take a clique $Q_{i}$ on $\ell+2$ vertices $q_{i}^{0}, \ldots, q_{i}^{\ell}, q_{i}^{\ell+1}$.

- For each edge $x y \in\left\{q_{i}^{k} q_{i}^{k+1}: 0 \leqslant k \leqslant \ell\right\}$ create an $S_{t-1}[x, y]$. No two of the $S_{t-1}$ have a vertex in common unless those in $\{x, y\}$.

- Take a chordless path on vertices $s_{i}^{0}, s_{i}^{1}, \ldots, s_{i}^{\lambda}$ and edges $s_{i}^{k} s_{i}^{k+1}, 0 \leqslant k<\lambda$.

- Connect each $s_{i}^{k}, 0 \leqslant k \leqslant \lambda$, to exactly $q_{i}^{0}$ and $q_{i}^{\ell+1}$.

- For each edges $x y \in\left\{s_{i}^{k} s_{i}^{k+1}: 0 \leqslant k<\lambda\right\}$ create an $S_{t-2}[x, y]$.

- For each edges $x y \in\left\{s_{i}^{0} q_{i}^{0}, s_{i}^{0} q_{i}^{\ell+1}, s_{i}^{\lambda} q_{i}^{0}, s_{i}^{\lambda} q_{i}^{\ell+1}\right\}$ create an $S_{t-(\ell+2)}[x, y]$.

Note that the clique $Q_{i}$ is a cutset of $G\left(v_{i}\right)$ and the components of $G\left(v_{i}\right)-Q_{i}$ are chordal. Thus, $G\left(v_{i}\right)$ is a chordal graph. See also Fig. 4.

For each clause $C_{j}$ create the graph $G\left(C_{j}\right)$ as follows. If $t$ is even, $G\left(C_{j}\right)$ is simply a single vertex $a_{j}$. If $t$ is odd, $G\left(C_{j}\right)$ is the graph $S_{t-1}\left[a_{j}^{1}, a_{j}^{2}\right]$. In any case, $G\left(C_{j}\right)$ is a chordal graph.

Finally, the graph $G=G(F)$ is obtained from all $G\left(v_{i}\right)$ and $G\left(C_{j}\right)$ by identifying all vertices $s_{i}^{0}$ to a single vertex $s$, and adding the following additional edges:

- connect every vertex in $Q_{i}$ with every vertex in $Q_{i^{\prime}}, i \neq i^{\prime}$. Thus, the cliques $Q_{i}$, $1 \leqslant i \leqslant n$, together form a clique $Q$ in $G$,

- for each literal $u_{i} \in\left\{q_{i}^{0}, q_{i}^{\ell+1}\right\}$, if $u_{i} \in C_{j}$ then connect $u_{i}$ with $a_{j}$ if $t$ is even, otherwise with $a_{j}^{1}$ and $a_{j}^{2}$.

The description of the graph $G=G(F)$ is complete. Clearly, $G$ can be constructed in polynomial time. 

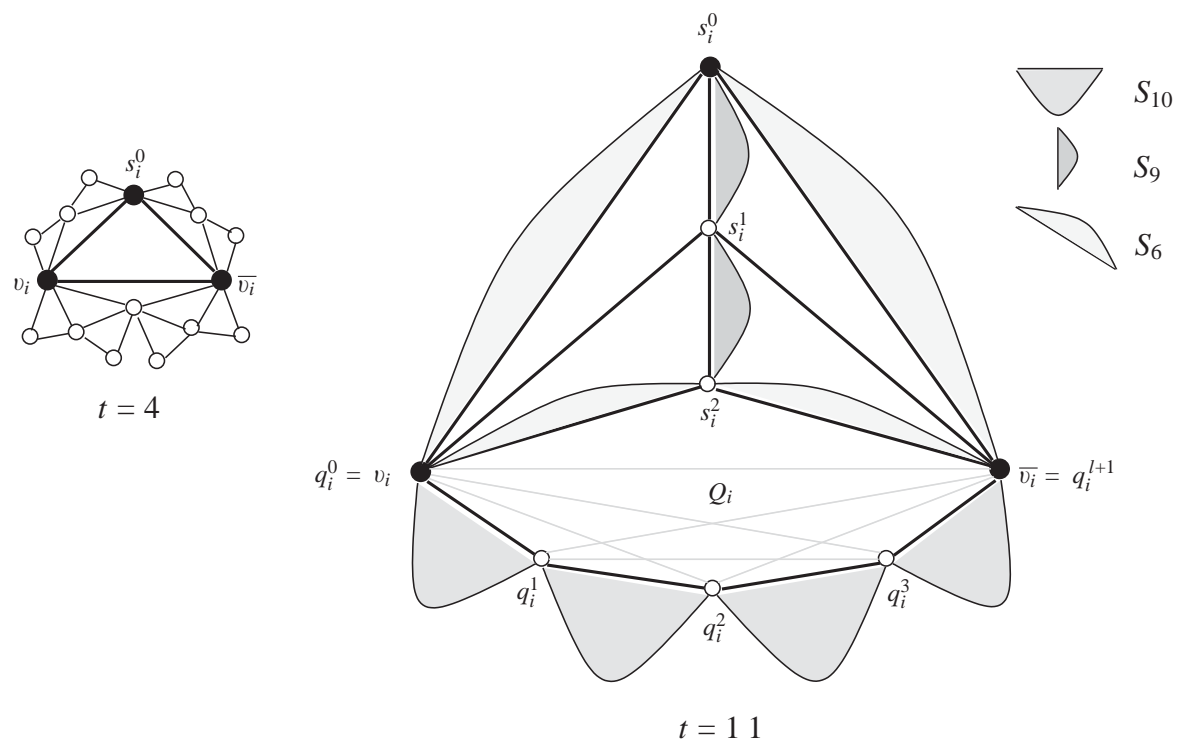

Fig. 4. The graph $G\left(v_{i}\right)$.

Lemma 1. G is chordal, and

$$
\operatorname{diam}(G) \leqslant \begin{cases}t+1 & \text { if } t \text { is even } \\ t+2 & \text { if } t \text { is odd. }\end{cases}
$$

Proof. The connected components $A$ of $G-Q$ are induced subgraphs of the chordal graphs $G\left(v_{i}\right)$ and $G\left(C_{j}\right)$, hence chordal. Since $Q$ is a clique and the graphs $G[Q \cup A]$ induced by $Q \cup A$ are chordal, $G$ is a chordal graph.

Consider two vertices $x, y$ of maximum distance in $G$. Then, by construction, we have the following cases.

- $x \in S_{t-2}\left[s_{i}^{k}, s_{i}^{k+1}\right], y \in S_{t-2}\left[s_{i^{\prime}}^{k^{\prime}}, s_{i^{\prime}}^{k^{\prime}+1}\right]$ for some $i \neq i^{\prime}$.

- $t$ is odd, and $x \in S_{t-2}\left[s_{i}^{k}, s_{i}^{k+1}\right], y \in G\left(C_{j}\right)$ for some $i, j$.

- $t$ is odd, and $x \in G\left(C_{j}\right), y \in G\left(C_{j^{\prime}}\right)$ for some $j \neq j^{\prime}$.

- $x \in S_{t-1}\left[q_{i}^{k}, q_{i}^{k+1}\right], y \in S_{t-1}\left[q_{i^{\prime}}^{k^{\prime}}, q_{i^{\prime}}^{k^{\prime}+1}\right]$ for some $i \neq i^{\prime}$.

By Observation 1(1) we have in the first case

$$
d(x, y) \leqslant 2 \cdot\left\lceil\frac{t-2}{2}\right\rceil+3= \begin{cases}t+1 & \text { if } t \text { even } \\ t+2 & \text { if } t \text { odd }\end{cases}
$$

in the second case,

$$
d(x, y) \leqslant\left\lceil\frac{t-2}{2}\right\rceil+\left\lceil\frac{t-1}{2}\right\rceil+3=t+2,
$$


in the third case,

$$
d(x, y) \leqslant 2 \cdot\left\lceil\frac{t-1}{2}\right\rceil+3=t+2,
$$

and in the last case (note that $Q$ is a clique),

$$
d(x, y) \leqslant 2 \cdot\left\lceil\frac{t-1}{2}\right\rceil+1 \leqslant t-1
$$

Lemma 2. Suppose $G$ admits a tree t-spanner. Then $F$ is satisfiable.

Proof. Let $T$ be a tree $t$-spanner of $G$.

Claim. For every $i$,

- all edges $q_{i}^{k} q_{i}^{k+1}, 0 \leqslant k \leqslant \ell$, belong to $T$, and

- exactly one of the edges $s q_{i}^{0}$ and $s q_{i}^{\ell+1}$ belongs to $T$.

Proof of the Claim. For every $0 \leqslant k \leqslant \ell$, we have by Observation 2 , the $q_{i}^{k} q_{i}^{k+1}$-path $F_{k}$ in $T$ must belong to the $S_{t-1}\left[q_{i}^{k}, q_{i}^{k+1}\right]$. Thus, $P=\bigcup_{k=0}^{\ell} F_{k}$ is the $q_{i}^{0} q_{i}^{\ell+1}$-path in $T$ and belongs to $\bigcup_{k=0}^{\ell} S_{t-1}\left[q_{i}^{k}, q_{i}^{k+1}\right]$. Therefore, as $T$ is a tree, $s q_{i}^{0}$ or $s q_{i}^{\ell+1}$ cannot belong to $T, s q_{i}^{0} \notin E(T)$, say.

If $s q_{i}^{\ell+1} \in E(T)$ then by Observation 2(1) applied on $S_{t-(\ell+2)}\left[s, q_{i}^{0}\right]$,

$$
\ell+2 \geqslant d_{T}\left(s, q_{i}^{0}\right)=|E(P)|+1 \geqslant \ell+2,
$$

hence $|E(P)|=\ell+1$ and the claim follows in this case.

Assume $s q_{i}^{\ell+1} \notin E(T)$. We will reach a contradiction. First, since $s q_{i}^{0}$ and $s q_{i}^{\ell+1}$ are not edges of $T$ and $s_{i}^{1}$ does not have any neighbor outside $G\left(v_{i}\right)$, Observation 2(1) shows that the $s s_{i}^{1}$-path in $T$ must belong to the $S_{t-2}\left[s, s_{i}^{1}\right]$. Then, neither $q_{i}^{0} s_{i}^{1}$ nor $q_{i}^{\ell+1} s_{i}^{1}$ belongs to $T$, because otherwise $d_{T}\left(s, q_{i}^{\ell+1}\right)>\ell+2$ or $d_{T}\left(s, q_{i}^{0}\right)>\ell+2$, respectively, contradicting Observation 2(1), applied on $S_{t-(\ell+2)}\left[s, q_{i}^{\ell+1}\right]$ and $S_{t-(\ell+2)}\left[s, q_{i}^{0}\right]$, respectively. Therefore, the $s_{i}^{1} s_{i}^{2}$-path in $T$ must belong to the $S_{t-2}\left[s_{i}^{1}, s_{i}^{2}\right]$. Continuing in this way we get

For $0 \leqslant k<\lambda$, the $s_{i}^{k} s_{i}^{k+1}$-path in $T$ belongs to the $S_{t-2}\left[s_{i}^{k} s_{i}^{k+1}\right]$.

Moreover, all edges $q_{i}^{0} s_{i}^{k}, q_{i}^{\ell+1} s_{i}^{k}(0 \leqslant k \leqslant \lambda)$ do not belong to $T$.

Now, consider the $s q_{i}^{0}$-path $R$ in $T$. Since $s q_{i}^{0}, s q_{i}^{\ell+1} \notin E(R), R$ cannot belong to the $S_{t-(\ell+2)}\left[s, q_{i}^{0}\right]$ or to the $S_{t-(\ell+2)}\left[s, q_{i}^{\ell+1}\right]$, otherwise $d_{T}\left(s, q_{i}^{\ell+1}\right) \geqslant(\ell+1)+|E(R)| \geqslant \ell+3$ or $d_{T}\left(s, q_{i}^{0}\right) \geqslant(\ell+1)+|E(R)| \geqslant \ell+3$, contradicting Observation 2 .

Furthermore, as each $s_{i}^{k}, 0<k \leqslant \lambda$, has no neighbor outside $G\left(v_{i}\right)$, there exists a $u_{i^{\prime}} \in\left\{q_{i^{\prime}}^{0}, q_{i^{\prime}}^{\ell+1}\right\}, i^{\prime} \neq i$, such that $u_{i^{\prime}}$ is the first vertex of $R$ that belongs to $Q$, starting from $s$. Let $r$ be the largest number such that the subpath of $P$ from $q_{i}^{0}$ to $q_{i}^{r}$ is a subpath of $R$. Without loss of generality, we may assume that $r \geqslant(\ell+1) / 2$, because either the $q_{i}^{0} s$-path of $T$ or the $q_{i}^{l+1} s$-path of $T$ uses at least half of $P$. 
Thus,

$$
d_{T}\left(q_{i}^{0}, s_{i}^{\lambda}\right) \geqslant r+d_{T}\left(q_{i}^{r}, u_{i^{\prime}}\right)+d_{T}\left(u_{i^{\prime}}, s\right)+\lambda \geqslant \frac{\ell+1}{2}+2+\left\lceil\frac{\ell}{2}\right\rceil .
$$

Also, by Observation 1(2), there exists an edge $u v \in S_{t-(\ell+2)}\left[q_{i}^{0}, s_{i}^{\lambda}\right]$ such that

$$
d_{T}(u, v) \geqslant d_{T}\left(q_{i}^{0}, s_{i}^{\lambda}\right)+t-(\ell+2),
$$

hence

$$
d_{T}(u, v) \geqslant \frac{\ell+1}{2}+2+\left\lceil\frac{\ell}{2}\right\rceil+t-(\ell+2)=t+\left\lceil\frac{\ell}{2}\right\rceil-\frac{\ell}{2}+\frac{1}{2}>t,
$$

a contradiction. The Claim follows.

Now, define a truth assignment $b$ for variables $v_{i}$ 's as follows:

$$
b\left(v_{i}\right)= \begin{cases}\text { true } & \text { if } s v_{i}=s q_{i}^{0} \in E(T), \\ \text { false } & \text { otherwise. }\end{cases}
$$

By the Claim, $b$ is well-defined. To see that $b(F)=$ true, assume to the contrary that there is some clause $C_{j}=\left(c_{j 1}, c_{j 2}, c_{j 3}\right)$ such that $b\left(c_{j 1}\right)=b\left(c_{j 2}\right)=b\left(c_{j 3}\right)=$ false. We distinguish two cases.

Case 1: $t$ is odd.

By definition of $b$, the edges $s c_{j 1}, s c_{j 2}$ and $s c_{j 3}$ do not belong to $T$. By the connectedness of $T$, there is exactly one edge in $T$ connecting $\left\{c_{j 1}, c_{j 2}, c_{j 3}\right\}$ and $\left\{a_{j}^{1}, a_{j}^{2}\right\}$. Without loss of generality, say $c_{j 1} a_{j}^{1} \in E(T)$.

By Observation 2, the $a_{j}^{1} a_{j}^{2}$-path in $T$ must belong to the $S_{t-1}\left[a_{j}^{1}, a_{j}^{2}\right]$. Thus, by the Claim and the fact that $s \bar{c}_{j k}, k=1,2,3$, are edges of $T$,

$$
\begin{aligned}
d_{T}\left(a_{j}^{2}, c_{j 2}\right) & =d_{T}\left(a_{j}^{2}, a_{j}^{1}\right)+1+d_{T}\left(c_{j 1}, \overline{c_{j 1}}\right)+2+d_{T}\left(\overline{c_{j 2}}, c_{j 2}\right) \\
& =d_{T}\left(a_{j}^{2}, a_{j}^{1}\right)+2(\ell+1)+3 \\
& \geqslant 2(\ell+1)+4=2\left(\left\lfloor\frac{t}{2}\right\rfloor-2+1\right)+4 \\
& =t+1,
\end{aligned}
$$

a contradiction because $T$ is a tree $t$-spanner of $G$.

Case 2: $t$ is even.

In this case, as $T$ is a tree, exactly one of the edges $c_{j 1} a_{j}, c_{j 2} a_{j}$ and $c_{j 3} a_{j}$ belongs to $T$, say $c_{j 1} a_{j} \in E(T)$. As in Case 1 we have:

$$
d_{T}\left(a_{j}, c_{j 2}\right)=2(\ell+1)+3=t+1,
$$

a contradiction.

Thus each clause $C_{j}$ of $F$ is satisfied by the assignment $b$, proving Lemma 2 .

Lemma 3. Suppose $F$ is satisfiable. Then $G$ admits a tree t-spanner. 


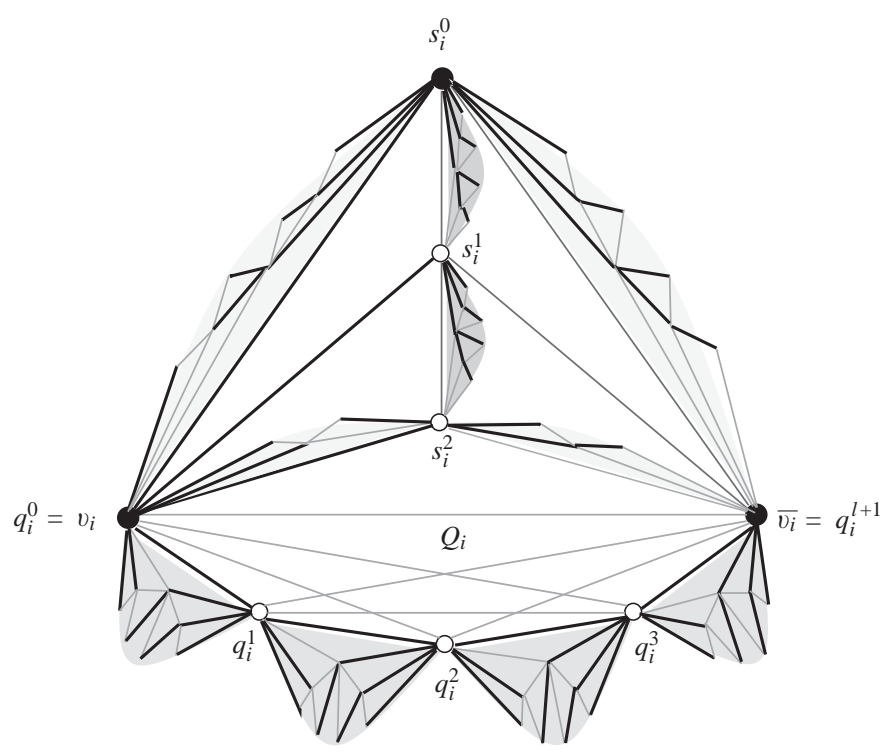

Fig. 5. The tree $t$-spanner $T_{i}$ (thick edges) in $G\left(v_{i}\right)$; illustrated in case $b\left(v_{i}\right)=$ true.

Proof. Let $b$ be a truth assignment for variables $v_{i}$ that satisfies $F$. We construct a spanning tree $T$ of $G$ as follows.

First, for each $i$, construct a tree $t$-spanner $T_{i}$ of $G\left(v_{i}\right)$ in the following way:

- For each $0 \leqslant k \leqslant \ell$, take a tree $t$-spanner in $S_{t-1}\left[q_{i}^{k}, q_{i}^{k+1}\right]$ containing the edge $q_{i}^{k} q_{i}^{k+1}$. Such a tree spanner exists by Observation 1.

- For each $0 \leqslant k<\lambda$, take a tree $(t-2)$-spanner in $S_{t-2}\left[s_{i}^{k}, s_{i}^{k+1}\right)$. Such a tree spanner exists by Observation 1(3).

- Let $u_{i} \in\left\{q_{i}^{0}, q_{i}^{\ell+1}\right\}$ such that $b\left(u_{i}\right)=$ true.

Take a tree $(t-(\ell+1))$-spanner in $S_{t-(\ell+2)}\left[s_{i}^{0}, u_{i}\right]$ containing the edge $s_{i}^{0} u_{i}$ into

$T_{i}$. Take a tree $(t-(\ell+1))$-spanner in $S_{t-(\ell+2)}\left[s_{i}^{\lambda}, u_{i}\right]$ containing the edge $s_{i}^{\lambda} u_{i}$ into $T_{i}$. By Observation 1, such tree spanners exist.

Take a tree $(t-(\ell+2))$-spanner in $S_{t-(\ell+2)}\left[s_{i}^{0}, \overline{u_{i}}\right)$ and a tree $(t-(\ell+2))$-spanner in $S_{t-(\ell+2)}\left[s_{i}^{\lambda}, \overline{u_{i}}\right)$ into $T_{i}$ which have the property given by Observation 1(3).

Add all edges $u_{i} s_{i}^{k}, 1 \leqslant k \leqslant \lambda-1$, into $T_{i}$. See Fig. 5 .

Claim 1. $T_{i}$ is a tree t-spanner of $G\left(v_{i}\right)$.

Proof of Claim 1. Let $u_{i} \in\left\{q_{i}^{0}, q_{i}^{\ell+1}\right\}$ such that $b\left(u_{i}\right)=$ true. By construction, we need only consider the following edges:

(a) Edges $s_{i}^{k+1} x$ in $S_{t-2}\left[s_{i}^{k}, s_{i}^{k+1}\right]$ where $x$ is any neighbor of $s_{i}^{k+1}$. Since the restriction of $T_{i}$ on $S_{t-2}\left[s_{i}^{k}, s_{i}^{k+1}\right)$ is a tree $(t-2)$-spanner and the edges $u_{i} s_{i}^{k}, u_{i} s_{i}^{k+1}$ belong to $T_{i}, d_{T_{i}}\left(s_{i}^{k+1}, x\right) \leqslant(t-2)+2=t$, and we are done.

(b) Edges $\overline{u_{i}} x$ in $S_{t-(\ell+2)}\left[s_{i}, \overline{u_{i}}\right] \cup S_{t-(\ell+2)}\left[s_{i}^{\lambda}, \overline{u_{i}}\right]$ where $x$ is any neighbor of $\overline{u_{i}}$. If $x \in\left\{s_{i}^{0}, s_{i}^{\lambda}\right\}, d_{T_{i}}\left(\overline{u_{i}}, x\right)=\ell+2=\left\lfloor\frac{t}{2}\right\rfloor<t$ and we are done. For $x \notin\left\{s_{i}^{0}, s_{i}^{\lambda}\right\}$ we have in 
case $x \in S_{t-(\ell+2)}\left[s_{i}^{0}, \overline{u_{i}}\right]$ (note that $s_{i}^{0} u_{i} \in E\left(T_{i}\right)$ ):

$$
\begin{aligned}
d_{T_{i}}\left(\overline{u_{i}}, x\right) & =d_{T_{i}}\left(\overline{u_{i}}, u_{i}\right)+1+d_{T_{i}}\left(s_{i}^{0}, x\right) \\
& \leqslant(\ell+1)+1+(t-(\ell+2))=t .
\end{aligned}
$$

The case $x \in S_{t-(\ell+2)}\left[s_{i}^{\lambda}, \overline{u_{i}}\right]$ is similar. Claim 1 follows.

Next, for each $j$, construct a tree $t$-spanner $T_{j}$ of $G\left(C_{j}\right)$ in the following way: If $t$ is odd, $T_{j}$ is a tree $t$-spanner in $G\left(C_{j}\right)$ containing the edge $a_{j}^{1} a_{j}^{2}$ (exists by Observation (1). If $t$ is even, $T_{j}$ is the single vertex $a_{j}$.

Now, to obtain the desired tree $t$-spanner $T$ of $G$ we identifying all $s_{i}^{0}$ in $T_{i}$ to the single vertex $s$, and for each $j$ connect $a_{j}^{1}$, respectively, $a_{j}$, according to the parity of $t$, to exactly one $u_{i} \in\left\{c_{j 1}, c_{j 2}, c_{j 3}\right\}$ such that $b\left(u_{i}\right)=$ true.

Claim 2. $T$ is a tree $t$-spanner of $G$.

Proof of Claim 2. By Claim 1 and the construction of $T$, we only need to consider edges $x y$ not incident with $s$ and with property

- $x \in Q_{i}, y \in Q_{i^{\prime}}$ for $i \neq i^{\prime}$, or

- $x \in\left\{a_{j}^{1}, a_{j}^{2}\right\}$ (if $t$ is odd), respectively, $x=a_{j}$ (if $t$ is even) and $y=c_{j k}$ for some $k \in\{1,2,3\}$ with $b\left(c_{j k}\right)=$ false.

Consider the first case. Let $u_{i} \in\left\{q_{i}^{0}, q_{i}^{\ell+1}\right\}$ and $u_{i^{\prime}} \in\left\{q_{i^{\prime}}^{0}, q_{i^{\prime}}^{\ell+1}\right\}$ such that $b\left(u_{i}\right)=b\left(u_{i^{\prime}}\right)$ $=$ false. By construction of $T$ we have

$$
\begin{aligned}
d_{T}(x, y) & \leqslant d_{T}\left(u_{i}, \overline{u_{i}}\right)+d_{T}\left(\overline{u_{i}}, s\right)+d_{T}\left(u_{i^{\prime}}, \overline{u_{i^{\prime}}}\right)+d_{T}\left(\overline{u_{i^{\prime}}}, s\right) \\
& =(\ell+1)+1+(\ell+1)+1=2\left\lfloor\frac{t}{2}\right\rfloor \\
& \leqslant t .
\end{aligned}
$$

In the second case, let $k^{\prime} \in\{1,2,3\}$ such that $b\left(c_{j k^{\prime}}\right)=$ true and such that $c_{j k^{\prime}} a_{j}^{1}$ (respectively, $c_{j k^{\prime}} a_{j}$ ) is an edge of $T$. Then, if $t$ is odd,

$$
\begin{aligned}
d_{T}(x, y) & \leqslant 1+d_{T}\left(a_{j}^{1}, c_{j k^{\prime}}\right)+d_{T}\left(c_{j k^{\prime}}, s\right)+d_{T}\left(s, \overline{c_{j k}}\right)+d_{T}\left(\overline{c_{j k}}, y\right) \\
& =4+(\ell+1)=3+\left\lfloor\frac{t}{2}\right\rfloor=3+\frac{t-1}{2} \\
& \leqslant t
\end{aligned}
$$

Similarly, if $t$ is even,

$$
d_{T}(x, y) \leqslant 3+(\ell+1)=2+\frac{t}{2} \leqslant t .
$$

The proof of Lemma 3 is complete.

Theorem 1 follows from Lemmas 1-3. We remark that the chordal graph $G$ constructed above always admits a tree $(t+1)$-spanner. 


\section{Tree spanners in chordal graphs of "smaller" diameter}

For a chordal graph $G$, it is known that $\operatorname{diam}(G) \geqslant 2 r(G)-2$ holds $[9,10]$, i.e., $r(G) \leqslant \operatorname{diam}(G) / 2+1$. This already yields the following.

Theorem 5. Let $t \geqslant 2$ be an even integer. Every graph $G$ of diameter at most $t-1$ admits a tree t-spanner. Moreover, if $G$ is in addition chordal, such a tree spanner can be constructed in linear time.

Proof. Choose a center vertex $z$ (i.e., the eccentricity of $z$ equals $r(G)$ ), and construct a Breadth-First-Search tree $T$ of $G$ rooted at $z$. By the choice of $z$ and as $T$ is a BFS-tree, we have for all edges $x y \in E(G)$ :

$$
\begin{aligned}
d_{T}(x, y) & \leqslant d_{T}(x, z)+d_{T}(y, z)=d_{G}(x, z)+d_{G}(y, z) \\
& \leqslant 2 r(G) \leqslant 2\left(\frac{t-2}{2}+1\right) \\
& =t
\end{aligned}
$$

because $r(G) \leqslant \operatorname{diam}(G) / 2 \leqslant((t-1) / 2)+1$, and, as $t$ is even, $r(G) \leqslant((t-2) / 2)+1$.

Note that a center vertex $z$ in a chordal graph can be detected in linear time [11]. So, the tree $T$ above can be constructed in linear time, too.

We remark that there are chordal graphs of diameter $t$ without tree $t$-spanner (consider for example $S_{2}[x, y]$ with an extra vertex $z$ adjacent to $x$ and $y$ only). Thus, Theorem 5 is best possible under diameter constraints.

Corollary 1. Every chordal graph of diameter at most 3 has a tree 4-spanner, where such a tree spanner can be constructed in linear time.

It remains an interesting open question whether existence of a tree 3-spanner in a given chordal graph of diameter at most 3 can be tested in polynomial time.

The proof of the following lemma is completely similar to the proof of Theorem 5 .

Lemma 4. Every graph $G$ admits a tree $(2 r(G))$-spanner. Moreover, if $G$ is in addition chordal, such a tree spanner can be constructed in linear time.

Let now $t$ be an odd integer $(t \geqslant 3)$. From Lemma 4 and the fact that $2 r(G) \geqslant \operatorname{diam}$ $(G) \geqslant 2 r(G)-2$ holds for any chordal graph $G$, we immediately deduce.

Theorem 6. Every chordal graph of diameter at most $t-2$ admits a tree $t$-spanner, and such a tree spanner can be constructed in linear time.

It would be nice to show also that, if $t \geqslant 3$ is an odd integer, then every chordal graph of diameter at most $t-1$ admits a tree $t$-spanner. But, although for chordal graphs 
with $\operatorname{diam}(G) \geqslant 2 r(G)-1$ this is true, it fails to hold for chordal graphs of diameter $2 r(G)-2$. There are even chordal graphs of diameter 2 without tree 3 -spanners. In what follows we will show that the existence of a tree $(2 r(G)-1)$-spanner in a chordal graph of diameter $2 r(G)-2$ "depends" on the existence of a tree 3-spanner in a chordal graph of diameter 2 .

First we present some auxiliary results. Let $v$ be a vertex of $G=(V, E)$. The disk centered at $v$ with radius $k$ is the set of all vertices having distance at most $k$ to $v$ : $D(v, k)=\left\{u: u \in V\right.$ and $\left.d_{G}(u, v) \leqslant k\right\}$. Let $r(v)$ be a non-negative integer associated with a vertex $v$ of a graph $G$. For a vertex set $M \subseteq V$, a subset $D \subseteq V$ is $r$-dominating for $M$ in $G$ if for all $v \in M$ there is a $u \in D$ with $d_{G}(u, v) \leqslant r(v)$. If the set $D$ $r$-dominates $M$ and is a clique then $D$ is an $r$-dominating clique for $M$ in $G$. The following simple necessary and sufficient condition for the existence of $r$-dominating cliques in chordal graphs was presented in [13].

Lemma 5. Let $G=(V, E)$ be a chordal graph, $M \subseteq V$ be any subset of $V$ and $r: M \rightarrow$ $\mathbf{N} \cup\{0\}$ be the radius function associated with $M$. Then $M$ has an $r$-dominating clique if and only if for every pair of vertices $v, w \in M$, the inequality

$$
d_{G}(v, w) \leqslant q r(v)+r(w)+1
$$

holds. Moreover, such a clique can be determined within time $O(|M| \cdot|E|)$.

A subset $S \subseteq V$ is m-convex if $S$ contains every vertex on every chordless path between vertices of $S$. We will need the following well-known property.

Lemma 6 (Farber and Jamison [15]). Any disk $D(v, k)$ of a chordal graph is m-convex.

For a subset $S \subseteq V$ and a vertex $v \in V$, let

$$
\operatorname{proj}(v, S)=\left\{u \in S: d_{G}(v, u)=d_{G}(v, S)\right\}
$$

be the metric projection of $v$ to $S$. For a subset $X \subseteq V$, let $\operatorname{proj}(X, S)=\bigcup_{v \in X}$ proj $(v, S)$. A subset $A \subseteq V$ is a $t w o$-set in $G$ if $d_{G}(v, u) \leqslant 2$ holds for every $v, u \in A$.

Lemma 7. Let $G$ be a (not necessarily chordal) graph. The metric projection proj $(A, S)$ of any two-set $A$ to an m-convex set $S$ of $G$ is a two-set.

Proof. Let $A$ be a two-set in $G$ and $u_{1}, u_{2}$ be two non-adjacent vertices from $\operatorname{proj}(A, S)$. Let also $v_{i}, i \in\{1,2\}$, be a vertex of $A$ with $u_{i} \in \operatorname{proj}\left(v_{i}, S\right)$ and $P_{i}$ be a shortest path between $u_{i}$ and $v_{i}$. Since $u_{i} \in \operatorname{proj}\left(v_{i}, S\right)$ no other vertex of $P_{i}$ belongs to $S$.

Let $u$ be any vertex in $D\left(v_{1}, 1\right) \cap D\left(v_{2}, 1\right)$. Let $H$ be the subgraph of $G$ induced by vertices in $V\left(P_{1}\right) \cup V\left(P_{2}\right) \cup\{u\}$. Then, let $P$ be any induced subpath of $H$ from $u_{1}$ to $u_{2}$. Since $u$ is the only vertex of $H \backslash\left\{u_{1}, u_{2}\right\}$ which may be in $S$, path $P$ is path $u_{1}, u, u_{2}$, because otherwise there is an induced $u_{1} u_{2}$-path in $G$ that contains at least one vertex not in $S$, which contradicts the fact that $S$ is m-convex. Thus, $d_{G}\left(u_{1}, u_{2}\right) \leqslant 2$. 
Lemma 8 (Chepoi [10] and Dragan and Brandstädt [13]). Let G be a (not necessarily chordal) graph. For any $m$-convex set $S$ and vertices $v \in V, u \in S$ of $G$ there is a vertex $w \in \operatorname{proj}(v, S)$ such that $d_{G}(v, u)=d_{G}(v, w)+d_{G}(w, u)$, i.e., $w$ lies on a shortest path between $v$ and $u$.

Lemma 9. In every chordal graph $G=(V, E)$ of diameter $2 r(G)-2$ there exists a two-set $S$ such that $d_{G}(v, S) \leqslant r(G)-2$ for every $v \in V$. Moreover such a two-set can be determined within time $O\left(|V|^{3}\right)$.

Proof. Assume that $\left(v_{1}, \ldots, v_{n}\right)$ is an ordering of $V$ and let $i$ be the largest index for which a two-set $S$ exists such that $d_{G}\left(v_{j}, S\right) \leqslant r(G)-2$ holds for every $j \in\{1, \ldots, i\}$. If $i<n$ then we have $d_{G}\left(v_{i+1}, S\right) \geqslant r(G)-1$.

First we show that $S$ can be chosen to be entirely in $D\left(v_{i+1}, r(G)\right)$. If $S \backslash D\left(v_{i+1}, r(G)\right) \neq \emptyset$, then consider the projection of $S$ to the set $D\left(v_{i+1}, r(G)\right)$. Due to the $m$-convexity of disks in chordal graphs, the projection $\operatorname{proj}\left(S, D\left(v_{i+1}, r(G)\right)\right.$ is a two-set, according to Lemma 7 . Denote this set by $S^{\prime}$. For all $j, j \leqslant i$, consider a vertex $u_{j} \in S \cap D\left(v_{j}, r(G)-2\right)$ and $w_{j} \in D\left(v_{j}, r(G)-2\right) \cap D\left(v_{i+1}, r(G)\right.$ ) (such vertices exist since $d_{G}\left(v_{j}, S\right) \leqslant r(G)-2$ and $\left.\operatorname{diam}(G)=2 r(G)-2\right)$. According to Lemma 8 there is a vertex $u_{j}^{\prime} \in S^{\prime}$ such that

$$
d_{G}\left(u_{j}, w_{j}\right)=d_{G}\left(u_{j}, u_{j}^{\prime}\right)+d_{G}\left(u_{j}^{\prime}, w_{j}\right)
$$

holds. Due to the $m$-convexity of disks, for all $j, j \leqslant i, u_{j}^{\prime} \in D\left(v_{j}, r(G)-2\right)$ is fulfilled, i.e., for all $j \leqslant i, d_{G}\left(v_{j}, S^{\prime}\right) \leqslant r(G)-2$. Thus, we could assume at the beginning that $S \subset D\left(v_{i+1}, r(G)\right)$.

Now we can apply Lemma 5 to the set $M=S \cup\left\{v_{i+1}\right\}$ with the radius function $r\left(v_{i+1}\right)=r(G)-2$ and $r(v)=1$ for all $v \in S$, and get a clique $C$ such that $d_{G}\left(v_{i+1}, C\right) \leqslant$ $r(G)-2$ and every vertex of $S$ is adjacent to a vertex of $C$. Let $u_{i+1}^{\prime}$ be a vertex of $C$ with $d_{G}\left(v_{i+1}, u_{i+1}^{\prime}\right) \leqslant r(G)-2$. It is easy to see that the set $A=S \cup\left\{u_{i+1}^{\prime}\right\}$ is a two-set and $d_{G}\left(v_{j}, A\right) \leqslant r(G)-2$ holds for any $j \leqslant i+1$. But this contradicts with the maximality of $i$. Thus, $i$ has to be equal to $n$ and the first part of the lemma follows.

Time bound: First we can determine within $O(|V| \cdot|E|)$ steps the distance matrix of $G$.

$i$-th iteration: Since the distances of $v_{i+1}$ to all other vertices are known in advance, the projection of two-set $S$ to the disk $D\left(v_{i+1}, r(G)\right)$ can be determined within $O(|V|$. $|S|)$ steps. The vertex $u_{i+1}^{\prime}$ can be determined also within $O\left(|V|^{2}\right)$ steps. There are at most $|V|$ such iterations, and each iteration requires at most $O\left(|V|^{2}\right)$ time.

Lemma 10. Every maximal by inclusion two-set of a chordal graph is m-convex.

Proof. Let $S$ be a maximal by inclusion two-set and assume that there is an induced path $P$ between vertices $x$ and $y$ such that $P \cap S=\{x, y\}$. Let $v$ be a vertex of $P$ adjacent to $x$. Since $v \notin S$ and $S$ is a maximal by inclusion two-set, there must be a vertex $w$ in $S$ with $d_{G}(v, w)=3, d_{G}(x, w)=2$ and $d_{G}(y, w) \leqslant 2$. Since $x, y \in D(w, 2)$ and vertex $v$ from induced path $P$ connecting $x$ and $y$ does not belong to $D(w, 2)$, a contradiction with $m$-convexity of disks in chordal graphs arises. 
Theorem 7. Chordal graphs of diameter $2 r(G)-2$ admit tree $(2 r(G)-1)$-spanners if and only if chordal graphs of diameter 2 admit tree 3-spanners. Moreover, if a tree 3-spanner of any chordal graph of diameter 2 can be found in polynomial time, then a tree $(2 r(G)-1)$-spanner of a chordal graph of diameter $2 r(G)-2$ can be found in polynomial time, too.

Proof. The "only if" direction is obvious.

The "if" direction. Let $G$ be a chordal graph of diameter $2 r(G)-2$. By Lemma 9, $G$ has a two-set $S$ such that $d_{G}(v, S) \leqslant r(G)-2$ holds for any $v \in V$, and such a set $S$ can be found in $O\left(|V|^{3}\right)$ time. We can extend $S$ to a maximal by inclusion two-set within the same time bound. So, without loss of generality, assume that $S$ is a maximal two-set and, hence, it is $m$-convex. Since $S$ contains together with any two vertices also all shortest paths connecting them in $G$, subgraph $G(S)$ of $G$ induced by the set $S$ is a chordal graph of diameter at most 2. By the theorem assumption, $G(S)$ has a tree 3-spanner $T(S)$. We can run a BFS on $G$ started at the set $S$ to extend the tree $T(S)$ to a spanning tree $T$ of $G$. We show now that $T$ is a $(2 r(G)-1)$-spanner for $G$. Consider any edge $x y \in E(G)$. Let $x^{\prime}$ and $y^{\prime}$ be the vertices of subtree $T(S)$ of a tree $T$ that are closest to $x$ and $y$ in $T$, respectively. Since $T$ is constructed from $T(S)$ (from $S$ ) in a BFS manner, we have $d_{T}\left(x, x^{\prime}\right)=d_{G}\left(x, x^{\prime}\right) \leqslant r(G)-2$ and $d_{T}\left(y, y^{\prime}\right)=d_{G}\left(y, y^{\prime}\right) \leqslant r(G)-2$. Let $P_{x}, P_{y}$ be the paths connecting $x$ with $x^{\prime}$ and $y$ with $y^{\prime}$ in $T$ and consider a path of $G$ formed by $P_{x}$, edge $x y$, and $P_{y}$. Since two nonadjacent vertices of $m$-convex set $S$ cannot be connected by a path with inner vertices outside $S$, we conclude that either $x^{\prime}=y^{\prime}$ or $x^{\prime} y^{\prime} \in E(G)$ and, hence, $x^{\prime} y^{\prime} \in E(G(S))$. Therefore, $d_{T}\left(x^{\prime}, y^{\prime}\right)=d_{T(S)}\left(x^{\prime}, y^{\prime}\right) \leqslant 3$ holds. Now, $d_{T}(x, y)=d_{T}\left(x, x^{\prime}\right)+d_{T}\left(x^{\prime}, y^{\prime}\right)+$ $d_{T}\left(y, y^{\prime}\right) \leqslant r(G)-2+3+r(G)-2=2 r(G)-1$ and we are done.

We do not know how to use this theorem for general chordal graphs (since not all chordal graphs of diameter 2 have tree 3 -spanners), but this theorem could be very useful for those hereditary subclasses of chordal graphs where each graph of diameter 2 is tree 3 -spanner admissible. Then, for every graph of diameter at most $t-1$ from those classes, a tree $t$-spanner will exist and it could be found in polynomial time if corresponding tree 3-spanner is constructable in polynomial time. For an arbitrary chordal graph $G$ with $\operatorname{diam}(G)=2 r(G)-2$, it can happen that a chordal graph of diameter at most 2, generated by a two-set of $G$ (found as described in Lemma 9 and Theorem 7), does not have a tree 3-spanner, but yet $G$ itself admits a $(2 r(G)-1)$ spanner. We are still working on TREE $(2 r(G)-1)$-SPANNER problem in chordal graphs of diameter $2 r(G)-2$. It is natural to ask whether a combination of Theorems 7 and 9 will work.

\section{Tree 3-spanners in chordal graphs of diameter 2}

In this section, we give an application of Theorem 7 as well as a criterion for the tree 3-spanner admissibility of chordal graphs of diameter at most 2. 
Lemma 11 (Chang and Nemhauser [9] and Voloshin [30]). Let $G$ be a chordal graph. If all vertices $v_{i}$ of a clique $C=\left\{v_{1}, \ldots, v_{k}\right\}$ have the same distance from a vertex $v \in V$ then there is a common neighbour $u$ of all elements of $C$ which has distance $d_{G}(v, C)-1$ to $v$.

This follows also from the necessary and sufficient condition for the existence of $r$-dominating cliques in chordal graphs. Consider $r(u)=0$ for all $u \in C$ and $r(v)=d_{G}$ $(v, C)-1$.

A graph $G$ is non-trivial if it has at least one edge.

Lemma 12. Let $G$ be a non-trivial chordal graph of diameter at most 2. If $G$ does not contain a clique $K_{5}$ on five vertices, then $G$ has a dominating edge, i.e., an edge $e \in E$ such that $d_{G}(v, e) \leqslant 1$ for any $v \in V$.

Proof. Notice that $G$ must have a dominating clique, i.e., a clique $C$ such that any vertex of $G$ is in $C$ or is adjacent to a vertex in $C$. For this it is enough to consider the neighborhood $N(v)$ of any simplicial vertex $v$ of $G$. Since $d_{G}(u, v) \leqslant 2$ for every $u \in V$, this clique $N(v)$ dominates $G$.

Consider now among all cliques dominating $G$ the one with minimum cardinality. Denote it also by $C$. We claim that $|C| \leqslant 2$. If this is not the case, then we can find three vertices $a, b, c$ in $C$ and three vertices $a^{\prime}, b^{\prime}, c^{\prime}$ outside $C$ with properties $N\left(a^{\prime}\right) \cap\{a, b, c\}=\{a\}, N\left(b^{\prime}\right) \cap\{a, b, c\}=\{b\}$ and $N\left(c^{\prime}\right) \cap\{a, b, c\}=\{c\}$. Vertices $a^{\prime}, b^{\prime}, c^{\prime}$ are called private neighbors of $a, b, c$, respectively. Since $G$ is chordal, vertices $a^{\prime}, b^{\prime}, c^{\prime}$ are pairwise non-adjacent.

First we show that then a vertex $v$ must exist such that $N(v) \supseteq\left\{a, b, c, a^{\prime}, b^{\prime}, c^{\prime}\right\}$. In fact, by chordality of $G$, it is enough to show that $N(v) \supseteq\left\{a^{\prime}, b^{\prime}, c^{\prime}\right\}$. Assume, by way of contradiction, that no such vertex $v$ exists. Since $\operatorname{diam}(G)=2$, there must be vertices $x, y, z$ in $G$ such that $x$ is adjacent to $a^{\prime}, c^{\prime}$ and not to $b^{\prime}, y$ is adjacent to $b^{\prime}, c^{\prime}$ and not to $a^{\prime}, z$ is adjacent to $a^{\prime}, b^{\prime}$ and not to $c^{\prime}$. By chordality of $G$, in cycle $a^{\prime}-x-c^{\prime}-y-b^{\prime}-z-a^{\prime}$ there must be chords $z x, x y, y z$. Analogously, by considering cycles on five vertices, we conclude that $x a, x c, y c, y b, z b, z a \in E$. Now, to avoid induced cycles on 4 vertices, two of the following three possible chords $z c, y a, x b$ must be present in $G$. In conclusion, at least five out of six vertices $\{a, b, c, x, y, z\}$ induce a complete subgraph in $G$, which is impossible.

Thus, there is a vertex $v$ in $G$ such that $N(v) \supseteq\left\{a, b, c, a^{\prime}, b^{\prime}, c^{\prime}\right\}$ for any triple $a^{\prime}, b^{\prime}, c^{\prime}$ of private neighbors of $a, b, c$. Next we show that any vertex $x \in V \backslash N(v)$ is adjacent to every vertex from $\{a, b, c\}$, thus concluding that $v t$ is a dominating edge of $G$ for any $t \in\{a, b, c\}$.

If $x$ is not adjacent to any vertex from $\{a, b, c\}$, then $d_{G}(x,\{a, b, c, v\})=2$ and we can apply Lemma 11 to get a new vertex $x^{\prime}$ which together with $a, b, c, v$ will induce a forbidden clique of size 5. Hence, $x$ must be adjacent to a vertex from $\{a, b, c\}$, say $x a \in E$. If now $x b, x c \notin E$ then $x$ is a private neighbor of $a$ and therefore there must be a vertex $u \neq v$ in $G$ such that $N(u) \supseteq\left\{a, b, c, x, b^{\prime}, c^{\prime}\right\}$. To avoid an induced cycle $b^{\prime}-u-c^{\prime}-v-b^{\prime}$, we must have a chord $u v$. But then $K_{5}=\{a, b, c, u, v\}$ arises. 
Assume now, without loss of generality, that $x a, x b \in E$ but $x c \notin E$. Then $x c^{\prime} \notin E$ since otherwise vertices $x, a, c, c^{\prime}$ would form an induced cycle. As $\operatorname{diam}(G)=2$, there is a common neighbor $u$ of $c^{\prime}$ and $x$ in $G$. By considering cycles $c^{\prime}-c-a-x-u-c^{\prime}$ and $c^{\prime}-c-b-x-u-c^{\prime}$ we get $u c, u b, u a \in E$. Also, to avoid an induced cycle $u-a-v-c^{\prime}-u$, a chord $u v$ must be present. Then again, a clique of size 5 on vertices $a, b, c, u, v$ arises. Hence, any vertex of $G$ not adjacent to $v$ is adjacent to all $a, b, c$.

Since neither planar graphs nor 3-trees have cliques on 5 vertices and any graph with a dominating edge is trivially tree 3 -spanner admissible, we conclude.

Corollary 2. Let $G$ be a non-trivial graph of diameter at most 2. If $G$ is a planar chordal graph or a $k$-tree for $k \leqslant 3$, then $G$ has a dominating edge and hence a tree 3-spanner.

As we mentioned in introduction, there is no constant $t$ such that planar chordal graphs or $k$-trees $(k \geqslant 2)$ are tree $t$-spanner admissible. So, it is interesting to mention the following result.

Theorem 8. Every chordal graph of diameter at most $t-1$, if it is planar or a $k$-tree $(k \leqslant 3)$, has a tree $t$-spanner and such a tree spanner can be constructed in polynomial time.

In what follows we will assume that $G$ is an arbitrary chordal graph which admits a tree 3-spanner $T$. Note that any tree of diameter at most 2 is a star and any tree of diameter 3 has a dominating edge (in this case $T$ is called a bistar).

Lemma 13. For any maximal (by inclusion) clique $C$ of a chordal graph $G$ one of the following conditions holds.

(a) $C$ induces a star in $T$,

(b) either $C$ induces a bistar in $T$ or there is a vertex $v \notin C$ such that $C \cup\{v\}$ induces a bistar in $T$.

Proof. Since $T$ is a 3 -spanner for $G$, for any two vertices $x, y$ of $C, d_{T}(x, y) \leqslant 3$ holds. If $T(C)$ is a subtree of $T$ (i.e., $T(C)$ is connected) then $T(C)$ is either a star or a bistar. If $T(C)$ is disconnected then, by Lemma 5 applied to $T$ and $C$, there must exist an edge $u v$ in $T$ which dominates all vertices of $C$ (in $T$ ). Assume that both vertices $u$ and $v$ lie outside $C$. Since $C$ is a maximal clique, there must be two vertices $v^{\prime}$ and $u^{\prime}$ in $C$ such that $v v^{\prime}, u u^{\prime} \in E(G)$ and $v u^{\prime}, u v^{\prime} \notin E(G)$. But then vertices $u, u^{\prime}, v^{\prime}, v$ form an induced cycle of length 4 in $G$, which is impossible.

Clearly, $T$ is a star only if $G$ has an universal vertex, and the diameter of $T$ is 3 only if $G$ has a dominating edge. The following theorem handles the case of all chordal graphs of diameter at most 2. Unfortunately, not every such graph has a dominating edge. There are chordal graphs of diameter 2 which do not have any tree 3 -spanners, 
and there are chordal graphs of diameter 2 that have a tree 3 -spanner but all those spanners are of diameter 4 .

Theorem 9. A chordal graph $G$ of diameter at most 2 admits a tree 3-spanner if and only if one of the following three conditions holds.

(1) $G$ has an universal vertex,

(2) $G$ has a dominating edge,

(3) there is a vertex $v$ in $G$ such that any connected component of the second neighborhood of $v$ has a dominating vertex in $N(v)$.

Proof (If direction). We construct a tree 3-spanner $T$ of $G$ in the following way. In case (1) we connect all vertices from $V \backslash\{v\}$, where $v$ is an universal vertex of $G$, to $v$. In case (2), for a dominating edge $u v$ of $G$, we connect all vertices of $N(v)$ to $v$ and all vertices from $V \backslash N(v)$ to $u$. In the last case, for that special vertex $v$, first we find all connected components of the second neighborhood $N_{2}(v)=\left\{w \in V: d_{G}(v, w)=2\right\}$ of $v$. Then for each component $A$ found, we search the set $N(v)$ for a vertex $a$ dominating $A$ and connect vertices of $A$ to $a$. Finally, we connect all vertices from $N(v)$ to $v$. It is easy to see that tree $T$ constructed in this way is a 3 -spanner of $G$.

(Only if direction) Assume that graph $G$ has a tree 3-spanner $T$ but neither of conditions (1) and (2) is fulfilled. Then the diameter of $T$ must be at least 4 . Consider vertices $a, b$ of $T$ at distance 4 and the middle vertex $v$ of a shortest path connecting $a$ with $b$. Let $S=\{x \in V: x v \in E(T)\}$ and $S^{\prime}=S \cup\{v\}$. Clearly, $|S| \geqslant 2$, and deleting vertices of $S^{\prime}$ from $T$ will disconnect $T$. Let $T_{a}$ and $T_{b}$ be subtrees of $T \backslash S^{\prime}$ containing vertices $a$ and $b$, respectively. Note that $T_{a}$ and $T_{b}$ are different subtrees of $T$. Since $T$ is a 3-spanner of $G$, no vertex from $V\left(T_{a}\right)$ is adjacent to a vertex of $V\left(T_{b}\right)$ in $G$. Hence, $S^{\prime}$ is a cutset of $G$. Moreover, from $\operatorname{diam}(G) \leqslant 2$ we deduce that every vertex of $G$ is either in $S^{\prime}$ or adjacent to a vertex of $S^{\prime}$. Therefore, any vertex from $N_{2}(v)$ (all neighborhoods here are considered in $G$ ) is adjacent in $G$ to a vertex of $S$. Consider now an arbitrary connected component $A$ of the subgraph of $G$ induced by set $N_{2}(v)$.

Claim 1. $N(A) \cap N(v)$ is a clique.

Proof. Consider any two vertices $t, s$ in $N(A) \cap N(v)$ and let $t^{\prime}$ and $s^{\prime}$ be neighbors in $A$ of $t$ and $s$, respectively, such that the distance $d_{G(A)}\left(t^{\prime}, s^{\prime}\right)$ is minimal. Let also $P$ be a path of length $d_{G(A)}\left(t^{\prime}, s^{\prime}\right)$ connecting $t^{\prime}$ and $s^{\prime}$ in $A$. If $t s \notin E(G)$, then this path $P$ together with vertices $t, s$ and $v$ will form an induced cycle of length greater than 3 in $G$, which is impossible.

Claim 2. Neighborhoods in $N(v)$ of any two adjacent vertices $s$ and $t$ from $A$ are comparable. That is, $N(t) \cap N(v) \subseteq N(s) \cap N(v)$ or $N(s) \cap N(v) \subseteq N(t) \cap N(v)$.

Proof. If those neighborhoods are non-comparable, then there must exist vertices $t^{\prime}$ and $s^{\prime}$ in $N(v)$ such that $t t^{\prime}, s s^{\prime} \in E(G)$ and $t s^{\prime}, s t^{\prime} \notin E(G)$. But then, since 
$t^{\prime} s^{\prime} \in E(G)$ (by Claim 1), vertices $t, s, s^{\prime}, t^{\prime}$ form an induced cycle of length 4 in $G$.

Claim 3. If there is a vertex $x$ in $A$ which is adjacent in $G$ to exactly one vertex of $S$, say $y$, then $A \subset N(y)$ must hold.

Proof. Consider any neighbor $u$ of $x$ in $A$. If $u y \notin E(G)$ then a neighbor $w$ of $u$ in $S$ must be adjacent to $x$ (by Claim (2) and a contradiction arises. Hence, $u y \in E(G)$ holds for any neighbor $u$ of $x$ in $A$.

Consider now a non-neighbor $v^{\prime}$ of $x$ in $A$ and assume that $v^{\prime} y \notin E(G)$. Then, $d_{G}\left(x, v^{\prime}\right)=d_{G}\left(y, v^{\prime}\right)=2$. Let $w$ be a neighbor of $v^{\prime}$ in $S$ and $u$ be a common neighbor of $x$ and $v^{\prime}$ ( $u$ can be either in $A$ or in $N(v) \backslash S$ ). Note that $w x \notin E(G)$. If $u \in N(v) \backslash S$ then vertices $y, u, w$ are pairwise adjacent by Claim 1 . If $u \in A$ then, $u y \in E(G)$ since $u$ is a neighbor of $x$ in $A$. Also, $y w \in E(G)$ by Claim 1 , and $u w \in E(G)$ by Claim 2 . Thus, in any case vertices $y, u, w$ are pairwise adjacent in $G$. Consider a maximal (by inclusion) clique $C_{y u w}$ containing vertices $y, u$ and $w$. Note that $C_{y u w}$ may contain $v$ if $u \in N(v)$. Two vertices of this clique, namely $y$ and $w$, are dominated in $T$ by vertex $v$. Recall that $y, w \in S$ and hence $y v, w v \in E(T)$. We have also that $v u \notin E(T)$ even if $v u \in E(G)$, since $u \notin S$. Hence, by Lemma 13, there is a vertex $z$ in $G$ such that $z v$ is the dominating edge for $C_{y u w}$ in $T$, i.e., $z v, z u \in E(T)$ (and hence $z \in S$ ).

Assume $z \neq y$ and consider a maximal (by inclusion) clique $C_{y x u}$ in $G$ containing vertices $y, x$ and $u$. Since vertices $y, u$ of $C_{y x u}$ are connected in $T$ by path $y-v-z-u$, the edge $v z$ must be the dominating edge of $C_{y x u}$ in $T$. But neither $v$ nor $z$ is adjacent to $x$. Hence, a contradiction with Lemma 13 arises.

Let now $z=y$ and $C_{w v^{\prime} u}$ be a maximal (by inclusion) clique of $G$ containing vertices $w, v^{\prime}$ and $u$. Since vertices $u, w$ of $C_{w v^{\prime} u}$ are connected in $T$ by path $u-y-v-w$, the edge $y v$ must be the dominating edge of $C_{w v^{\prime} u}$ in $T$. But again neither $y$ nor $v$ is adjacent to $v^{\prime}$, and a contradiction with Lemma 13 arises.

So, contradictions obtained show that any non-neighbor $v^{\prime}$ of $x$ in $A$ must be adjacent to $y$, too. Therefore, $A \subset N(y)$, and Claim 3 follows.

By Claim 3 we may assume now that any vertex of $A$ has at least two neighbors in $S$. Also, by Claim 1, those neighbors have to be adjacent. Consider any edge $u x$ of $A$. We claim that there exists a vertex $z \in S$ such that $z x, z u \in E(T)$ holds.

Since the neighborhoods of $u$ and $x$ in $S$ have to be comparable, there must be two vertices $w$ and $y$ in $S$ such that $u, x, w$ and $y$ together form a clique in $G$. Let $C_{x y u w}$ be a maximal (by inclusion) clique of $G$ containing all four vertices $x, y, u, w$. Two vertices of this clique, namely $y$ and $w$, are dominated in $T$ by vertex $v$ since $y, w \in S$. We have also that $v u, v x \notin E(T)$ since $v u, v x \notin E(G)$. Then, by Lemma 13, there must be a vertex $z$ in $G$ such that $z v$ is the dominating edge for $C_{x y u w}$ in $T$, i.e., $z v, z u, z x \in E(T)$ (and therefore $z \in S$ ).

Thus, end-vertices of any edge of $A$ are dominated in $T$ by one vertex from $S$. Since all vertices of $S$ are adjacent in $T$ to $v$ and $T$ cannot contain any cycles, there must be just one vertex in $S$ that dominates in $T$ (and hence in $G$ ) all vertices of $A$. 
Since conditions (1) and (2) are particular cases of condition (3) we have the following.

Corollary 3. A chordal graph $G$ of diameter at most 2 admits a tree 3-spanner if and only if there is a vertex $v$ in $G$ such that any connected component of the second neighborhood of $v$ has a dominating vertex in $N(v)$.

We conclude this section with the following corollary.

Corollary 4. For a given chordal graphs $G=(V, E)$ of diameter at most 2, TREE 3-SpANNER can be decided in $O(|V||E|)$ time. Moreover, a tree 3-spanner of $G$, if it exists, can be constructed within the same time bound.

Proof. First we can search $G$ for an universal vertex in $O(|E|)$ time, and for a dominating edge or/and for a special vertex $v$ with the property described in Theorem 9 (condition 3) in $O(|V||E|$ ) time. Then the construction of $T$ (see the proof of Theorem 9) will take only linear time.

\section{Tree spanners in strongly chordal graphs}

For an integer $k \geqslant 3$, a $k$-sun consists of a $k$-clique $\left\{v_{1}, \ldots, v_{k}\right\}$ and a $k$-vertex stable set $\left\{u_{1}, \ldots, u_{k}\right\}$, and edges $u_{i} v_{i}, u_{i} v_{i+1}, 1 \leqslant i<k$, and $u_{k} v_{k}, u_{k} v_{1}$. A chordal graph is strongly chordal [14] if it does not contain a $k$-sun as an induced subgraph. In [3], it is proved that every strongly chordal graph admits a tree 4-spanner and such a tree spanner can be constructed in linear time. Not every strongly chordal graph has a tree 3-spanner. Actually, Tree 3-SPANNER remains open on strongly chordal graphs.

A $k$-planet is obtained from a $k$-path $v_{1} v_{2} v_{3} \cdots v_{k}$ and a triangle $a b c$ by adding edges $a v_{i}, 1 \leqslant i \leqslant k-1$ and $b v_{i}, 2 \leqslant i \leqslant k$; see Fig. 6 .

Definition 1. A chordal graph is called very strongly chordal if and only if it does not contain a $k$-planet as an induced subgraph.

As a 3 -sun is a 3-planet and every $k$-sun $(k \geqslant 4)$ contains an induced 4-planet, the class of very strongly chordal graphs is properly contained in the class of strongly chordal graphs. Moreover, the class of very strongly chordal graphs contains all interval

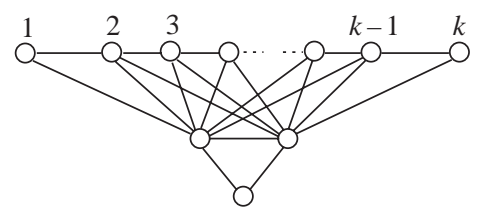

Fig. 6. A $k$-planet. 
graphs and all distance hereditary chordal graphs, called ptolemaic graphs [9]. The nice feature of this subclass of strongly chordal graphs is

Theorem 10. Every very strongly chordal graph admits a tree 3-spanner and such a tree spanner can be constructed in linear time.

Proof. Let $v$ be an arbitrary vertex of $G$. Write

$$
N_{i}(v)=\{x: d(v, x)=i\},
$$

and assume that $V(G)=\bigcup_{i=0}^{q} N_{i}(v)$. Let $G_{i}=G\left[N_{i}(v)\right]$, the subgraph of $G$ induced by $N_{i}(v)$.

Claim. For every $i=1, \ldots, q$ and for every connected component $A$ of $G_{i}$ there exists a vertex $v_{A} \in N_{i-1}(v)$ adjacent to all vertices in $A$.

Proof of the Claim. Assume that the claim is false, and consider the smallest $i$ such that the statement does not hold. Then $i>1$ and there exist two vertices $u_{1}, u_{2} \in B:=$ $N(A) \cap N_{i-1}(v)$ such that

$$
N_{A}\left(u_{1}\right) \backslash N_{A}\left(u_{2}\right) \neq \emptyset \quad \text { and } \quad N_{A}\left(u_{2}\right) \backslash N_{A}\left(u_{1}\right) \neq \emptyset .
$$

Fix two such vertices $u_{1}, u_{2}$ and choose $a \in N_{A}\left(u_{1}\right) \backslash N_{A}\left(u_{2}\right)$ and $a^{\prime} \in N_{A}\left(u_{2}\right) \backslash N_{A}\left(u_{1}\right)$ such that the shortest $a a^{\prime}$-path in $A$ has minimum length. Note that, as $G$ is chordal, $B$ is a clique, and hence, by the minimality of $i$, there exists a vertex $u \in N_{i-2}(v)$ adjacent to $u_{1}$ and $u_{2}$. Now, let $a_{1} a_{2} \cdots a_{k}$ be the shortest $a a^{\prime}$-path in $A ; a_{1}:=a$ and $a_{k}:=a^{\prime}$. As $G$ is chordal, $k \geqslant 3$ and each $a_{j}$ must be adjacent to $u_{1}$ or to $u_{2}$. By the choice of $a$ and $a^{\prime}$, each $a_{j}, 1<j<k$, must be adjacent to both $u_{1}$ and $u_{2}$. But then $a_{1}, \ldots, a_{k}$, $u_{1}, u_{2}$, and $u$ induce a $k$-planet, a contradiction. The claim is proved.

The claim shows that the following procedure constructs a tree 3 -spanner $T$ of $G$ correctly:

1. $V(T):=V(G) ; E(T):=\emptyset$

2. for $i:=q$ downto 1 do

3. for each connected component $A$ of $G_{i}$ do

4. $\quad$ choose a vertex $v_{A} \in N_{i-1}(v)$

5. $\quad E(T):=E(T) \cup\left\{v_{A} x: x \in A\right\}$

The running time of this procedure is at most

$$
\begin{aligned}
\sum_{i=1}^{q} O\left(\left|V\left(G_{i}\right)\right|+\left|E\left(G_{i}\right)\right|\right) & =O\left(\sum_{i=1}^{q}\left(\left|V\left(G_{i}\right)\right|+\left|E\left(G_{i}\right)\right|\right)\right. \\
& =O(|V(G)|+|E(G)|) .
\end{aligned}
$$

Another well-known subclass of strongly chordal graphs consists of the intersection graphs of directed paths in a rooted directed tree, called directed path graphs. The class 
of directed path graphs generalizes interval graphs naturally, and contains all ptolemaic graphs [12], and is tree 3-spanner admissible [21].

The intersection graphs of paths in a tree are called (undirected) path graph. We call shortly a graph strongly path graph if it is strongly chordal as well as a path graph. Clearly, every directed path graph is a strongly path graph, but not vice versa. Indeed, there are many strongly path graphs having no tree 3-spanner (while every directed path graph does [21]). Moreover, in contrast to strongly chordal graphs, for every $t$, there is a path graph having no tree $t$-spanner [20].

\section{Tree spanners in $k$-split graphs}

A split graph is one whose vertex set can be partitioned into a clique and a stable set. Split graphs are exactly those chordal graphs whose complements are chordal as well. It is known (and easy to see; cf. $[6,20,29]$ ) that every split graph admits a tree 3 -spanner. We are going to describe a new subclass of chordal graphs containing all split graphs and still are tree 3-spanner admissible.

First, for an arbitrary graph $G$ let $S(G)$ be the set of all simplicial vertices of $G$. We also use $S(G)$ for the subgraph of $G$ induced by $S(G)$.

Lemma 14. For every connected component $A$ of $S(G)$,

(1) $A$ is a clique,

(2) every vertex in $A$ is adjacent to every vertex in $N(A)$,

(3) $N(A)$ is a clique.

Proof. (1) If $x \neq y$ are two non-adjacent vertices in $A$ then there exists a chordless $x y$-path $x z_{1} z_{2} \cdots z_{k} y$ in $A, k \geqslant 1$. Then, as $x$ and $z_{2}$ (possibly $z_{2}=y$ ) are non-adjacent, $z_{1}$ cannot be a simplicial vertex of $G$.

(2) If $v \in N(A)$ is non-adjacent to a vertex in $A$, then by (1), any neighbor of $v$ in $A$ would be a non-simplicial vertex.

(3) By (2), every two vertices in $N(A)$ have a common neighbor in $A$, hence the are adjacent because vertices in $A$ are simplicial vertices in $G$.

Lemma 15. If $G \backslash S(G)$ has a tree $(t-1)$-spanner then $G$ has a tree t-spanner.

Proof. Let $T^{\prime}$ be a tree $(t-1)$-spanner in $G \backslash S(G)$. According to Lemma 14(2) we construct a spanning tree $T$ of $G$ as follows.

1. $V(T):=V(G) ; E(T):=E\left(T^{\prime}\right)$

2. for each connected component $A$ of $S(G)$ do

3. choose a vertex $v_{A} \in N(A)$

4. $\quad E(T):=E(T) \cup\left\{v_{A} x: x \in A\right\}$

$T$ is a tree $t$-spanner of $G$ : Consider an edge $x y$ of $G$. If $x y$ belongs to $G \backslash S(G)$, $d_{T}(x, y)=d_{T^{\prime}}(x, y) \leqslant t-1$. If $x y$ belongs to $S(G), d_{T}(x, y)=2$ by construction. If 
$x \in G \backslash S(G)$ and $y \in S(G)$, say $x \in N(A), y \in A$ for some connected component $A$ of $S(G)$, then

$$
d_{T}(x, y)=1+d_{T}\left(v_{A}, x\right)=1+d_{T^{\prime}}\left(v_{A}, x\right) \leqslant 1+(t-1)=t .
$$

Note that, given $T^{\prime}$ and $S(G)$, the tree $t$-spanner $T$ of $G$ can be constructed in linear time.

Definition 2. For an arbitrary graph $G$ and an integer $k \geqslant 0$ let $G_{k}:=G_{k-1} \backslash S\left(G_{k-1}\right)$; $G_{0}:=G$. A graph $G$ is called $k$-split if $G_{k}$ is a clique.

Clearly, 0 -split graphs are exactly the cliques, and all split graphs are 1-split but not vice versa. The following fact is probably known.

Proposition 2. A graph $G$ is chordal if and only if $G$ is $k$-split for some $k$.

Proof. Since every chordal graph has a simplicial vertex, the if-part is obvious. Assume now that $G_{k}$ is a clique for some $k$. Then by Lemma 14, the graph $G_{k-1}$ (induced by $G_{k}$ and $S\left(G_{k-1}\right)$ ) is chordal. Repeating this argument we get that $G=G_{0}$ is chordal, too.

Theorem 11. Every $k$-split graph admits a tree $(k+2)$-spanner.

Proof. Since $G_{k}$ is a clique, it admits a tree 2-spanner. It follows from Lemma 15 that $G=G_{0}$ admits a tree $(k+2)$-spanner.

Corollary 5. All 1-split graphs, hence all split graphs, admit a tree 3-spanner, and a such a tree 3-spanner can be constructed in linear time, given the set of all simplicial vertices.

Note that the existence of a tree $(k+2)$-spanner in $k$-split graphs is best possible: there are many $k$-split graphs without tree $(k+1)$-spanner; for example, the 3 -sun is 1 -split (even split) and has no tree 2-spanner.

\section{Conclusion}

In this paper we have proved that, for any $t \geqslant 4$, TREe $t$-SpanNer is NP-complete on chordal graphs of diameter at most $t+1$ (if $t$ is even), respectively, at most $t+2$ (if $t$ is odd), improving the hardness result in [7] on a restricted well-understood graph class. We have shown that every chordal graph $G$ of diameter at most $t-1$ is tree $t$-spanner admissible if $\operatorname{diam}(G) \neq 2 r(G)-2$.

The complexity of TREE $t$-SPANNER remains unresolved on chordal graphs of diameter $t$ (if $t$ is even) and of diameter $t$ or $t+1$ (if $t$ is odd). Tree $t$-Spanner remains also open on path graphs and the case $t=3$ remains even open on path graphs that are strongly chordal graphs as well. However, we have shown that all very strongly chordal graphs, 
a subclass of strongly chordal graphs that contains all interval graphs and all ptolemaic graphs, are tree 3-spanner admissible, and a tree 3-spanner for a given very strongly chordal graph can be constructed in linear time. This improves known results on tree 3 -spanners in interval graphs [20,22,27]. We have also improved known results on tree 3 -spanners in split graphs $[6,20,29]$ by showing that all 1 -split graphs, a subclass of chordal graphs containing all split graphs, are tree 3-spanner admissible, and a tree 3-spanner for a 1-split graph can be constructed in linear time, given the set of its simplicial vertices. We presented a polynomial time algorithm for the TREE 3-SPANNER problem on chordal graphs of diameter at most 2.

Many questions remain still open. Among them:

(1) Can Tree 3-Spanner be decided efficiently on chordal or strongly chordal graphs? At least for strongly path graphs? And on 2-split graphs?

(2) Can Tree $(2 r(G)-1)$-SPANNER be decided efficiently on chordal graphs of diameter $2 r(G)-2$ ?

(3) What is the complexity of Tree $t$-SPANNER for chordal graphs of diameter at most $t$ ?

We also do not know whether all very strongly chordal graphs are strongly path graphs. Are these graphs even directed path graphs?

\section{Acknowledgements}

We wish to acknowledge the anonymous referees for suggestions leading to improvements in the presentation.

\section{References}

[1] B. Awerbuch, A. Baratz, D. Peleg, Efficient broadcast and light-weighted spanners, manuscript, 1992.

[2] H.-J. Bandelt, A. Dress, Reconstructing the shape of a tree from observed dissimilarity data, Adv. Appl. Math. 7 (1986) 309-343.

[3] A. Brandstädt, V. Chepoi, F. Dragan, Distance approximating trees for chordal and dually chordal graphs, J. Algorithms 30 (1999) 166-184.

[4] A. Brandstädt, V.B. Le, J. Spinrad, Graph Classes: A Survey, SIAM Monographs on Discrete Mathematics and Applications SIAM, Philadelphia, 1999.

[5] P. Buneman, A characterization of rigid circuit graphs, Discrete Math. 9 (1974) 48-50.

[6] L. Cai, Tree spanners: spanning trees that approximate the distances, Ph.D. Thesis, University of Toronto, 1992.

[7] L. Cai, D.G. Corneil, Tree spanners: an overview, Congressus Numer. 88 (1992) 65-76.

[8] L. Cai, D.G. Corneil, Tree spanners, SIAM J. Discrete. Math. 8 (1995) 359-387.

[9] G.J. Chang, G.L. Nemhauser, The $k$-domination and $k$-stability problems on sun-free chordal graphs, SIAM. J. Alg. Disc. Meth. 5 (1984) 332-345.

[10] V.D. Chepoi, Centers of triangulated graphs, Math. Notes 43 (1988) 82-86.

[11] V.D. Chepoi, F.F. Dragan, Linear-time algorithm for finding a center vertex of a chordal graph, Lecture Notes in Computer Science, Vol. 855, Springer, Berlin, 1994, pp. 159-170.

[12] E. Dahlhaus, Chordale Graphen in besonderem Hinblick auf parallele Algorithmen, Habilitation Thesis, Universität Bonn, 1991.

[13] F.F. Dragan, A. Brandstädt, $r$-Dominating cliques in Helly graphs and chordal graphs, Proc. of the 11th STACS, Caen, France, Springer, Lecture Notes in Computer Science, Vol. 775, Springer, Berlin, 1994, pp. 735-746; Discrete Math. 162 (1996) 93-108. 
[14] M. Farber, Characterizations of strongly chordal graphs, Discrete Math. 43 (1983) 173-189.

[15] M. Farber, R.E. Jamison, Convexity in graphs and hypergraphs, SIAM J. Alg. Discr. Meth. 7 (1986) 433-444.

[16] S.P. Fekete, J. Kremer, Tree spanners in planar graphs, Discrete Appl. Math. 108 (2001) 85-103.

[17] F. Gavril, The intersection graphs of subtrees in trees are exactly the chordal graphs, J. Combin. Theory (B) 16 (1974) 47-56.

[18] M.C. Golumbic, Algorithmic Graph Theory and Perfect Graphs, Academic Press, New York, 1980.

[19] Hoàng-Oanh Le, Effiziente Algorithmen für Baumspanner in chordalen Graphen, Diploma Thesis, Department of Mathematics, Technical University of Berlin, 1994.

[20] H.-O Le, V.B. Le, Optimal tree 3-spanners in directed path graphs, Networks 34 (1999) 81-87.

[21] E. Howorka, A characterization of ptolemaic graphs, J. Graph Theory 5 (1981) 323-331.

[22] M.S. Madanlal, G. Venkatesan, C. Pandu Rangan, Tree 3-spanners on interval, permutation and regular bipartite graphs, Inform. Process. Lett. 59 (1996) 97-102.

[23] I.E. Papoutsakis, Two structure theorems on tree spanners, M.Sc. Thesis, Department of Computer Science, University of Toronto, 1999.

[24] I.E. Papoutsakis, On the union of two tree spanners of a graph, Sixth Internat. Conf. on Graphs Theory Marseilles, France, August 28-September 1, 2000.

[25] D. Peleg, Distributed Computing: A Locality-Sensitive Approach, SIAM Monographs on Discrete Mathematics and Application, SIAM, Philadelphia, 2000.

[26] D. Peleg, A. Schaeffer, Graph spanners, J. Graph Theory 13 (1989) 99-116.

[27] E. Prisner, Distance approximating spanning trees, in: Proc. STACS'97, Lecture Notes in Computer Science, Vol. 1200, Springer, Berlin, 1997, pp. 99-510.

[28] J. Soares, Graph spanners: a survey, Congressus Numer. 89 (1992) 225-238.

[29] G. Venkatesan, U. Rotics, M.S. Madanlal, J.A. Makowsky, C. Pandu Ragan, Restrictions of minimum spanner problems, Inform. Comput. 136 (1997) 143-164.

[30] V.I. Voloshin, On properties of triangulated graphs, Oper. Res. Prog. (Kishinev), (1982) 24-32, (in Russian).

[31] J.R. Walter, Representations of rigid cycle graphs, Ph.D. Thesis, Wayne State University, Detroit, 1972. 